\title{
Wir müssen umdenken ...
}

eder Einzelne - auch der medizinische Laie - kann leicht nachvollziehen, dass eine vermiedene Krankheit nicht nur einen Vorteil subjektiven Wohlbefindens in sich birgt, sondern auch die geringsten Kosten verursacht. Da der weit überwiegende Anteil unserer medizinischen Leistungen „selbst verschuldetes Elend“ betrifft, müsste man konsequenterweise vordringlich die Einsicht in dieses Selbstverständnis wecken, um die finanziellen Aufwendungen und Belastungen in unserem Gesundheitssystem eindämmen zu können. Nur eine Umstellung und Kontrolle der persönlichen Lebensweise bewirkt ein höheres Maß an Lebensqualität und ungestörtem Wohlbefinden. Dies ist leicht gesagt - doch warum fällt uns das so schwer?

Die Zusammenhänge von Folgen der Lebensweise wie Übergewicht, mangelnde Bewegung, Suchtverhalten (Alkohol, Nikotin, Schleckereien und andere mehr) mit den allbekannten Krankheiten des Herzens, des Kreislaufs und seiner Regulierungsqualität, den Gefäßen, der anatomischen und mechanischen Funktionen sind unstrittig evident. Auch die Auswirkung der Eindämmung dieser Erkrankungen und deren Todesfolgen auf die finanziellen Belastungen der mediko-sozialen Systeme sind - individuell und epidemiologisch - überzeugend belegt. Schon in den letzten Jahrzehnten des ausgehenden letzten Jahrhunderts wurden beispielsweise in Finnland erhebliche präventivmedizinische Programme umgesetzt, die nicht nur Folgekrankheiten wie Diabetes mellitus und koronare Herzerkrankungen überzeugend reduzierten, sondern nachweislich das Gesundheitssystem erheblich entlasteten.

Die größten Schwierigkeiten, diesen Änderungsprozess zu bewirken, liegen jedoch nicht im Mangel geltender gesetzlicher Instrumente und Rahmenbedingungen. Vielmehr ist es die übermächtige Verführung der Lebens- und Umweltbedingungen unserer im Vergleich zur Dritten Welt paradiesisch abgesicherten materiellen Existenz mit einem Versorgungsüberfluss jenseits jeden Maßes biologischer Bedürftigkeit. Das so genannte „Fast Food“ beispielsweise ist nicht nur so verlockend, weil es durch den zum Teil extrem hohen Fettgehalt (billiger Überschussabfall aus der „Tierproduktion“) die beigefügten Geschmackstoffe (Gewürze u.a.) besonders zur Wirkung kommen lässt, sondern durch seine vordergründige Bequemlichkeit (Tisch decken, Abwaschen, Esskultur). Folgen dieser Verhaltens- und Ernährungsweisen zeigen sich schon früh in der zunehmenden extremen Fettleibigkeit mit allen internistischen, orthopädischen und geistigen Trägheitsfolgen.

Allein schon wegen des zahlenmäßigen Anstiegs dieser Erkrankungen wird eine Finanzierung im herkömmlichen traditionellen Verfahren der Gesundheitssysteme nicht mehr möglich sein. Vielleicht wird uns dieser Prozess zwingen, den Stellenwert von Präventionsmaßnahmen im ärztlichen Leistungsspektrum wieder zu er- höhen, um damit auch mit einer adäquaten Honorierung der ärztlichen Leistung einen weiteren Schub für eine Änderung der Verhaltensnormen der Menschen zu bewirken. So schrecklich die Auswirkungen auf die finanziellen Belastungen der Gesundheitssysteme sind, liegt die Paradoxie der heutigen Situation darin, dass bei klar erkannter Ursache keine konsequenten Maßnahmen zur Korrektur von eindeutig negativen Ernährungskonzepten ergriffen werden.

Es bedarf eines sehr frühen Ansatzes bereits im Kindergarten, um den erforderlichen Grundstock zu legen, der eine Neubewertung der eigenen Lebensbefindlichkeit ermöglicht. Wahrscheinlich wird dies jedoch ein verlorener Kampf sein, da eine übermächtige industrielle Organisation die Fast-Food-Mentalität weiter fördert. Denn diese weiß sehr wohl, welche Maßnahmen den Markt erhalten, stabilisieren oder gar ausweiten. Wir Mediziner müssen kategorisch erklären, dass die Prävention, also die Vermeidung von Krankheit, der einzig wirksame Ansatz ist, mit dem finanziellen Dilemma der medizinischen Korrektur selbstverschuldeten Elends erfolgreich umzugehen.

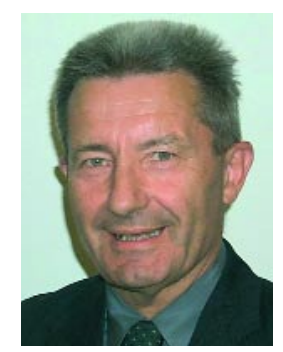

Prof. Dr. Dr. Dr. A. Grünert, Ulm

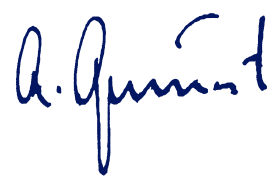

